\title{
ON EXTENSIONS OF $H$-SPACES
}

\author{
BY \\ JAMES DILLON STASHEFF(1)
}

1. Introduction. The following is known:

THEOREM A [2]. Let $Y$ be a space with only two nonvanishing homotopy groups, $\pi_{p}(Y)=\pi, \pi_{q}(Y)=G, q>p$. Y admits a multiplication if and only if the $k$-invariant $k \in H^{q+1}(\pi, p ; G)$ is primitive.

By primitive we mean $m^{*}(k)=p_{1}^{*}(k)+p_{2}^{*}(k)$ where $m: K \times K \rightarrow K$ is the multiplication on $K=K(\pi, p)$ (there is only one up to homotopy) and $p_{i}: K_{1} \times K_{2} \rightarrow K_{i}$ are the projections. Since $k=f^{*}(\iota)$ for some map $f: K(\pi, p) \rightarrow K(G, q+1)$, we can rephrase primitivity in terms of the map $f$. Using obstruction theory, we find that $k$ is primitive if and only if $f$ is an $H$-map, in the sense of:

Definition 1. Let $(X, m)$ and $(W, n)$ be $H$-spaces. A map $f: X \rightarrow W$ is an $H$-map if there exists a sputnik homotopy $h_{t}: X \times X \rightarrow W$ such that

$$
\begin{aligned}
& h_{0}(x, y)=f(x) f(y), \quad h_{1}(x, y)=f(x y), \quad \text { i.e., }
\end{aligned}
$$

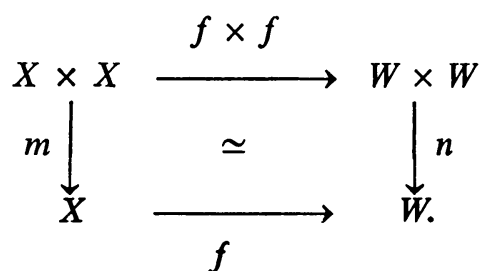

In the present paper, we wish to examine a somewhat more general situation more fully with four goals in mind:

(1) to give multiplications on $Y$ explicitly in terms of a sputnik homotopy for a map representing $k$,

(2) to examine more general conditions under which it is possible to define a multiplication in this way,

(3) to relate these multiplications to additive secondary cohomology operations,

(4) to extend the results to homotopy associative $H$-spaces.

We are indebted to J. C. Moore for suggesting these problems and for super-

Presented to the Society January 22, 1959 under the title On homotopy associativity of H-Spaces. Preliminary Report; received by the editors September 22, 1961.

(1) The author is a C.L.E. Moore Instructor. This research was supported in part by an NSF pre-doctoral fellowship and in part by the United States Air Force under contract No. AF49 (638)-42, monitored by the Air Force Office of Scientific Research of the Air Force Research and Development Command. 
vising the thesis which grew out of them [8]. Our viewpoint is a direct descendent of one which he presented in a seminar on related problems [7]. A very similar approach, though disguised by different notation, is used by Hilton [3] to prove Theorem 2.

As for our own notation, most of it is standard but a few things may need explanation. If $X$ and $Y$ are spaces with basepoints $e_{x}$ and $e_{y}$ respectively, then $X \wedge Y$ is the space $X \times Y / X \times e_{y} \cup e_{x} \times Y$.

In a diagram, (C) indicates commutavity of the portion surrounding it, while $\simeq$ indicates homotopy commutativity.

Let $X^{I}$ be the space of maps of the unit interval $I$ into $X$ and let $R$ be the set of non-negative real numbers. The (Moore) free path space $\mathscr{P}(X)$ is the space of all pairs $(f, r)$ where $r \in R$ and $f:[0, r] \rightarrow X$, topologized so that the map $h: \mathscr{P}(X) \rightarrow X^{I} \times R$, given by $h(f, r)=\left(f^{\prime}, r\right)$ with $f^{\prime}(t)=f(r t), 0 \leqq t \leqq 1$, is a homeomorphism onto its image $[5 ; 1]$. Paths $(f, r)$ and $(g, s)$ such that $f(r)=g(0)$ are added by the rule $(f, r)+(q, s)=(h, r+s)$ where

$$
\begin{array}{ll}
h(t)=f(t), & 0 \leqq t \leqq r, \\
h(t)=g(t-r), & r \leqq t \leqq r+s .
\end{array}
$$

The (Moore) based path space $\mathscr{L} X$ is the subspace of $\mathscr{P}(X)$ consisting of paths $(f, r)$ such that $f(0)=e_{x}$. The map $\pi: \mathscr{L} X \rightarrow X$ given by $\pi(f, r)=f(r)$ is a fibre map with fibre $\Omega X$, the (Moore) space of loops on $X$. We will often write $f$ for $(f, r)$ since a function uniquely determines its own domain of definition and, when convenient, will write $\overline{1}$ for $r$. Thus we write $\pi(f)=(f(\overline{1}))$. With the rule of addition mentioned above, $\Omega X$ becomes an associative $H$-space.

An $H$-space $(X, m)$ consists of a space $X$ with basepoint $e$ and a multiplication $m: X \times X \rightarrow X$ such that $m(x, e)=m(e, x)=x$.

If $(X, m)$ is an $H$-space, a multiplication $\mathscr{P} m: \mathscr{P} X \times \mathscr{P} X \rightarrow \mathscr{P} X$ can be defined by

$$
\begin{aligned}
\mathscr{P}_{m}[(f, r),(g, s)](t) & =m(f(t), g(t)) \text { for } t \leqq \min [r, s], \\
& =m(f(t), g(s)) \text { if } \min [r, s]=s \leqq t \leqq r, \\
& =m(f(r), g(t)) \text { if } \min [r, s]=r \leqq t \leqq s .
\end{aligned}
$$

The unit is $\lambda_{e}:[0,0] \rightarrow e$.

2. Explicit multiplications. We turn to the achievement of our first goal.

THEOREM 2. Let $(X, m)$ and $(W, n)$ be $H$-spaces. Let the fibring $\Omega W \rightarrow Y \stackrel{\mathfrak{p}}{\rightarrow} X$ be induced from $\Omega W \rightarrow \mathscr{L} W \rightarrow W$ by a map $f: X \rightarrow W$. If $f$ is an H-map, then there exists a multiplication $s: Y \times Y \rightarrow Y$ such that

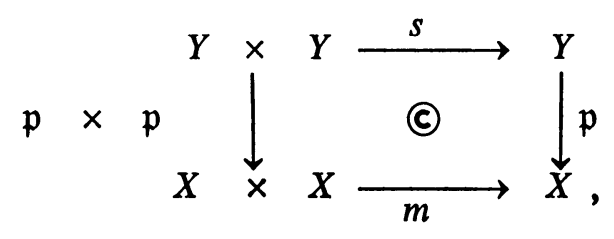




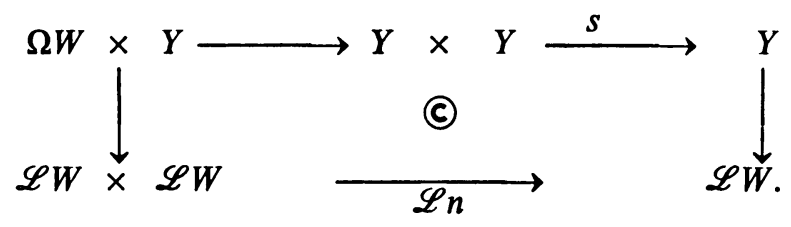

Proof. $Y$ can be represented as $\{(x, \lambda) \mid x \in X, \lambda \in \mathscr{L} W, \pi(\lambda)=f(x)\}$. If $f$ is an $H$-map, we have a homotopy $h_{t}: X \times X \rightarrow W$ such that $h_{0}(x, y)=f(x) f(y)$ and $h_{1}(x, y)=f(x y)$. Consider $\mathscr{P}(W)$, the space of all maps $\lambda:[0, r] \rightarrow W$. Corresponding to $h_{t}$, there is a map $F^{1}: X \times X \rightarrow \mathscr{P}(W)$ such that $F^{1}(x, y)(t)$ $=h_{t}(x, y)$. According to $[4$, Corollary (4.4)] we can assume without loss of generality that $h_{t}(x, e)=h_{t}(e, x)=f(x)$ for all $t$. If $F^{1}$ corresponds to such a homotopy, we can deform it (through maps $F^{s}: X \times X \rightarrow \mathscr{P}(W)$ such that $F^{s}(x, y)(0)=f(x) f(y)$ and $\left.F^{s}(x, y)(\overline{1})=f(x y)\right)$ to a map $F: X \times X \rightarrow \mathscr{P}(W)$ such that $F(x, e)=F(e, x)$ is the path of length 0 and value $f(x)$. We call $F$ a homotopy multiplier for $f$.

Now define $s$ by

$$
s\left[(x, \lambda),\left(x^{\prime}, \lambda^{\prime}\right)\right]=\left(x x^{\prime}, \lambda \lambda^{\prime}+F\left(x, x^{\prime}\right)\right) .
$$

We see that $s$ is well defined, i.e., $\pi\left(\lambda \lambda^{\prime}+F\left(x, x^{\prime}\right)\right)=f\left(x x^{\prime}\right)$, and has $\left(e, \lambda_{e}\right)$ as a unit. Diagrams (2.1) and (2.2) are clearly commutative. ( $F^{1}$ would have done as good a job if we didn't require (2) and were satisfied with a homotopy unit.)

In certain situations, this is the only way that $Y$ can have a multiplication.

THEOREM 3. Let $Y$ be as in Theorem 2. If there exists a multiplication satisfying (2.1), then $f$ is an $H$-map provided that $X$ is $(p-1)$-connected and for some $q \geqq p, \pi_{i}(W)=0$ for $i \leqq q$ and $i>p+q$.

Proof. The obstructions to $f$ being an $H$-map would be classes in $H^{i}\left(X \wedge X ; \pi_{i}(W)\right)$. Since $\mathfrak{p}$ is multiplicative, $(\mathfrak{p} \wedge \mathfrak{p})^{*}$ would take them into the corresponding obstructions for $f \mathfrak{p}$. These must all vanish since $f \mathfrak{p}$ is null-homotopic. However, $(\mathfrak{p} \wedge \mathfrak{p})^{*}$ is an isomorphism since $\pi_{i}(W)=0$ for $i>p+q$ and $\mathfrak{p}^{*}: H^{i}(X) \rightarrow H^{i}(Y)$ is an isomorphism for $i \leqq q$. Thus all the obstructions vanish; $f$ is an $H$-map. (Note that, up to homotopy, $W$ admits only one multiplication.)

THeOReM 4. Let $Y$ be as in Theorem 2. If $Y$ admits a multiplication s', then $f$ is an $H$-map with respect to some multiplication on $X$ provided $X$ is $(p-1)$-connected and for some $q \geqq p, \pi_{i}(X)=0$ for $i>2 q$ while $\pi_{i}(W)=0$ for $i \leqq q$ and $i>p+q$.

Proof. Since $\pi_{i}(W)=0$ for $\mathrm{i} \leqq q$, we can construct a cross-section $\chi: X^{q} \rightarrow Y$. Define a map $m^{\prime}$ by the following diagram:

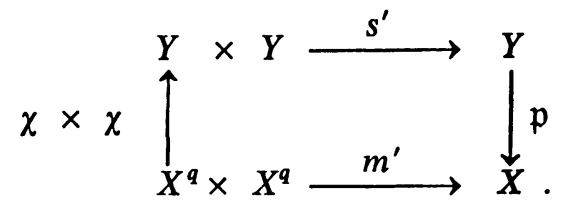


The obstructions to extending $m^{\prime}$ to all of $X \times X$ lie in $H^{i+1}\left(X \wedge X, X^{q} \wedge X^{q} ; \pi_{i}(X)\right)$ and hence all vanish since $\pi_{i}(X)=0$ for $i+1 \geqq 2 q+2$. Let $m: X \times X \rightarrow X$ be an extension of $m^{\prime}$. The obstructions to $\mathfrak{p}$ being an $H$-map with respect to $s^{\prime}$ and $m$ lie in $H^{i}\left(Y \wedge Y, Y^{q} \wedge Y^{q} ; \pi_{i}(X)\right)$ which again are trivial groups. Since $\mathfrak{p}$ is a fibring, if $\mathfrak{p} s^{\prime}$ is homotopic to $m(\mathfrak{p} \times \mathfrak{p})$, then $s^{\prime}$ can be deformed to a multiplication $s$ such that $p s=m(\mathfrak{p} \times \mathfrak{p})$. Thus Theorem 3 applies; $f$ must be an $H$-map.

Combining Theorems 2 and 4 yields Theorem A. More generally, we can say:

THEOREM 5. A space $Y$ admits a multiplication if and only if each stage $Y_{q}$ of the Postnikov system of $Y$ admits a multiplication $m_{q}: Y_{q} \times Y_{q} \rightarrow Y_{q}$ such that

(1) the projection $Y_{q+1} \rightarrow Y_{q}$ is multiplicative, and

(2) the k-invariant $k_{q+1} \in H^{q+2}\left(Y_{q} ; \pi_{q+1}(Y)\right)$ is primitive with respect to $m_{q}$.

3. Additive cohomology operations and $H$-spaces. Theorem 5 fails to be a completely satisfactory solution of our problem in the following sense:

In order to test $k_{q+1}$ for primitivity, we must specify $m_{q}$. Now in light of Theorems 2 and $3, m_{q}$ corresponds to a chain $b \in C^{q}\left(Y_{q-1} \wedge Y_{q-1} ; \pi_{q}(Y)\right)$ such that $\delta b=m_{q-1}^{*}(a)-p_{1}^{*}(a)-p_{2}^{*}(a)$ where $p_{i}, i=1,2$ are the projections and $a$ represents $k_{q}$. Given such a cochain $b$, how we can tell if $k_{q+1}$ is primitive with respect to the corresponding multiplication? We lack the technique for a general answer to this question, but in certain interesting cases the following approach is possible:

A cohomology class in $H^{q+1}(\pi, p ; G)$ can also be regarded as a primary cohomology operation. Theorem A then says that $Y$ admits a multiplication if and only if the $k$-invariant is an additive primary operation. It is this point of view which yields a neat generalization. Frank Adams [0] has defined secondary cohomology operations in terms of fibrings with fibre and base being abelian groups, while Spanier has suggested a somewhat different point of view. We have both these approaches in mind as we proceed.

Following [7], we generalize our notion of cohomology by replacing maps into $K(\pi, n)$ by maps into abelian topological groups (which have the homotopy type of products of $K(\pi, n)$ 's). Given an abelian topological group $A$, for $n \geqq 0$ denote by $\Omega^{n} A$ an abelian group whose underlying space has the indicated homotopy type. For $n>0$, denote $\Omega^{-n} A$ an abelian group $B$ such that $\Omega^{n} B \simeq A$. We define $H^{n}(X ; A)$ as $\pi\left(X, \Omega^{n} A\right)$, the group of homotopy classes of $X$ into $\Omega^{n} A$, with the obvious addition. We abbreviate $H^{0}(X ; A)$ as $H(X ; A)$. Using obstruction theory, it is easy to show that a universally defined cohomology operation $\mathcal{O}: H^{n}(; A) \rightarrow H^{m}(; B)$ corresponds to an element of $H^{m}\left(\Omega^{n} A ; B\right)$, i.e. to a homotopy class of maps of $\Omega^{n} A$ into $\Omega^{m} B$. We can also show that $\mathcal{O}$ is additive if and only if it is represented by an $H$-map.

Now let $A, B, C$ be abelian groups and $\mathcal{O}: H(; A) \rightarrow H(; B), \mathscr{N}: H(; B)$ $\rightarrow H(; C)$ be operations such that $\mathscr{N} \mathcal{O}=0$. 
DeFINITION 6. The rough secondary operation

$$
(\mathcal{O}, \mathscr{N}): \operatorname{Ker} \mathcal{O} \cap H^{0}(X ; A) \rightarrow H^{-1}(X ; C) / \Omega \mathscr{N} \circ H^{-1}(X ; B)+H^{-1}(A ; C) \circ[u]
$$

is defined as follows: Let $f: A \rightarrow B$ and $g: B \rightarrow C$ represent $\mathcal{O}$ and $\mathscr{N}$ respectively, so that $g f \simeq 0$. Let $\Omega B \rightarrow Y \rightarrow A$ be the fibre space over $A$ induced by $f$ from $\Omega B \rightarrow \mathscr{L} B \rightarrow B$. Let $h: Y \rightarrow \Omega C$ be any map such that $h \mid \Omega B \simeq \Omega g$. Now let $u: X \rightarrow A$ be any map representing a class in the kernel of $\mathcal{O}$. Since $f u \simeq 0, f u$ can be lifted to $\bar{u}: X \rightarrow Y$. The coset $(\mathcal{O}, \mathscr{N})[u]$ is represented by $h \bar{u}$.

DEFINITION 7. Given $f$ and $g$ representing $\mathcal{O}$ and $\mathscr{N}$ and a particular lifting $j: A \rightarrow \mathscr{L C}$ of $g f$, the precise secondary operation $(\mathcal{O}, \mathscr{N}, j): \operatorname{Ker} \mathcal{O} \rightarrow \operatorname{Coker} \Omega \mathscr{N}$ is defined as follows: Let $\Omega C \rightarrow D \rightarrow B$ be the fibre space induced by $g$. Define $h: Y \rightarrow \Omega C$ by $h(x, \lambda)=\mathscr{L} g(\lambda)-j(x)$ for $x \in A, \lambda \in \mathscr{L} B, \lambda(\overline{1})=f(x)$, observing that $h \mid \Omega B=\Omega g$. Given $\bar{u}: X \rightarrow Y$ lifting $u: X \rightarrow A$ (which represents a class in the kernel of $\mathcal{O}$ ), the $\operatorname{coset}(\mathcal{O}, \mathscr{N}, j)[u]$ is represented by $h \bar{u}$. (Notice that we obtain the same coset if we let $j$ vary through homotopic liftings, i.e., there is a homotopy $j_{t}: A \rightarrow \mathscr{L} C$ such that $j_{t}(x)(\overline{1})=g f(x)$ for all $t$.)

Definition 8. A precise secondary operation $(\mathcal{O}, \mathscr{N}, j)$ is additive if $\mathcal{O}$ is additive and $(\mathcal{O}, \mathscr{N}, j)[u+v]=(\mathcal{O}, \mathscr{N}, j)[u]+(\mathcal{O}, \mathscr{N}, j)[v]$ for any two classes $[u],[v]$ in $\operatorname{Ker} \mathcal{O}$. A similar definition applies to rough operations.

THEOREM 9. A precise secondary operation $(\mathcal{O}, \mathcal{N}, j)$ is additive if and only if there exists a multiplication son $Y$ such that $\mathfrak{p}: Y \rightarrow A$ is multiplicative and $h$ is an $H$-map.

Proof. Suppose there exists such a multiplication on $Y$. Let $u, v: X \rightarrow A$ be in the kernel of $\mathcal{O}$ so that $f u \simeq 0 \simeq f v$. Let $\bar{u}, \bar{v}: X \rightarrow Y$ be liftings of $u$ and $v$ respectively, then $q(\bar{u} \times \bar{v})$ is a lifting of $u+v$. Now $(\mathcal{O}, \mathscr{N}, j)[u+v]$ is represented by $h q(\bar{u} \times \bar{v})$ which is homotopic to $h(\bar{u})+h(\bar{v})$, a representative of $(\mathcal{O}, \mathscr{N}, j)[u]+$ $(\mathcal{O}, \mathcal{N}, j)[v]$, i.e., $(\mathcal{O}, \mathcal{N}, j)$ is additive.

Conversely, suppose $(\mathcal{O}, \mathscr{N}, j)$ is additive and let $u=v=\mathfrak{p}: Y \rightarrow A$. Since $\mathcal{O}$ is additive, $f$ is an $H$-map and by Theorem 2, $Y$ admits some multiplication $s^{\prime}: Y \times Y \rightarrow Y$ such that $\mathfrak{p}$ is multiplicative. Since $h$ itself represents $(\mathcal{O}, \mathscr{N}, j)[u]$ and $h s^{\prime}$ represents $(\mathcal{O}, \mathscr{N}, j)[u+u]$, the homotopy class of $h+h$ must differ from that of $h s^{\prime}$ by a class in the indeterminacy. Thus we can write $h+h \simeq h s^{\prime}+(\Omega \mathscr{N}) \phi$ for some map $\phi: Y \times Y \rightarrow \Omega B$. Define $s: Y \times Y \rightarrow Y$ by $s=s^{\prime}+\phi$ where +denotes the usual action of $\Omega B$ on $T$, corresponding to that of $\Omega B$ on $\mathscr{L} B$. Thus we have $h+h \simeq h s$ which proves the theorem.

COROLLARY 10. Let $(\mathcal{O}, \mathscr{N}, j)$ be a precise secondary operation represented by the map $h: Y \rightarrow \Omega C$. Let $\Omega^{2} C \rightarrow Z \rightarrow Y$ be induced by $h$. For the space $Z$ to admit a multiplication, it is sufficient for $(\mathcal{O}, \mathscr{N}, j)$ to be additive. The condition is necessary if for some $p \leqq q \leqq r$ we have

$$
\begin{array}{ll}
\pi_{i}(A)=0 & \text { for } i<p \quad \text { and } i>2 q, \\
\pi_{i}(B)=0 & \text { for } i<q+1 \text { and } i>p+q, \\
\pi_{i}(C)=0 & \text { for } i<r+2 \text { and } i>2 q .
\end{array}
$$


To apply Theorem 9 , can we determine the additivity of $(\mathcal{O}, \mathscr{N}, j)$ without constructing $Y$ or its multiplication $s$ ?

Definition 11. Given $H$-maps $f: U \rightarrow V, g: V \rightarrow W$ with homotopy multipliers $F: U \times U \rightarrow \mathscr{P}(V)$ and $G: V \times V \rightarrow \mathscr{P}(W)$, the composite homotopy multiplier $(G, F)$ for $g f$ is defined by

$$
(G, F)\left(u_{1}, u_{2}\right)=G\left(f\left(u_{1}\right), f\left(u_{2}\right)\right)+(\mathscr{P} g) F\left(u_{1}, u_{2}\right) .
$$

THEOREM 12. Let a precise secondary operation $(\mathcal{O}, \mathscr{N}, j)$ be determined by $f, g$ and $j$ as above. $(\mathcal{O}, \mathscr{N}, j)$ is additive if

(1) $f$ and $g$ are $H$-maps (i.e., $\mathcal{O}$ and $\mathscr{N}$ are additive) with homotopy multipliers $F$ and $G$, and

(2) $j$ is an H-map with a homotopy multiplier $J$ which covers the composite homotopy multiplier $(G, F)$.

The converse is true if for some $p \leqq q \leqq r$

$$
\begin{aligned}
& \pi_{i}(A)=0 \text { for } i<p \text { and } i>2 q \text {, } \\
& \pi_{i}(B)=0 \text { for } i<q+1 \text { and } i>p+q \text {, } \\
& \pi_{i}(C)=0 \text { for } i<r+2 \text { and } i>p+q+1 \text {. }
\end{aligned}
$$

Proof. Suppose $f, g$ and $j, F, G$, and $J$ are as above. Define a multiplication on $Y$ as in Theorem 2, i.e., for $x_{1}, x_{2} \in A, \lambda_{1}, \lambda_{2} \in \mathscr{L} B$ with $\lambda_{i}(\overline{1})=f\left(x_{i}\right)$, we have

Thus

$$
\left(x_{1}, \lambda_{1}\right)\left(x_{2}, \lambda_{2}\right)=\left(x_{1} x_{2}, \lambda_{1} \lambda_{2}+F\left(x_{1}, x_{2}\right)\right) \text {. }
$$

$$
h\left(\left(x_{1}, \lambda_{1}\right)\left(x_{2}, \lambda_{2}\right)\right)=\mathscr{L} g\left(\lambda_{1} \lambda_{2}\right)+(\mathscr{P} g) F\left(x_{1}, x_{2}\right)-j\left(x_{1} x_{2}\right)
$$

while

$$
h\left(x_{1}, \lambda_{1}\right) h\left(x_{2}, \lambda_{2}\right)=\mathscr{L} g\left(\lambda_{1}\right) \mathscr{L} g\left(\lambda_{2}\right)-j\left(x_{1}\right) j\left(x_{2}\right) .
$$

A sputnik homotopy for $h$ is described by the following diagram, the homotopy parameter running vertically and the loop parameter horizontally:

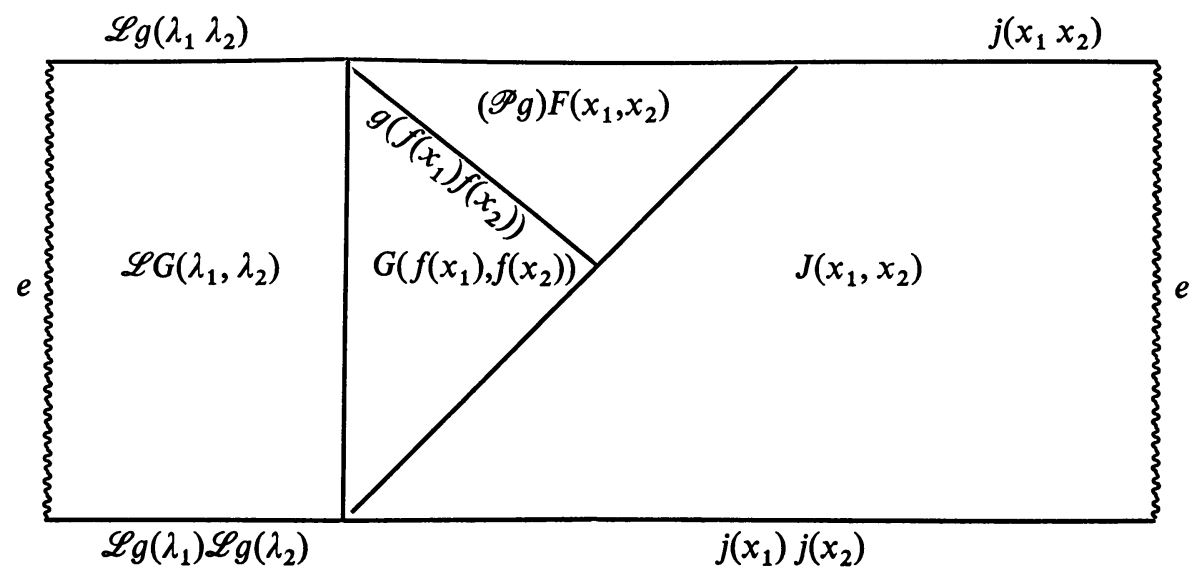

where the right hand trapezoid can be filled in by $J$. 
As for the converse, consider the space $Z$ induced over $Y$ by $h$. It is the same as the space induced over $A$ by $\psi: A \rightarrow D$ defined by $\psi(x)=(f(x), j(x))$. Since $\pi_{i}(C)=0$ for $i>p+q+1<2 q+1$, [g] is a loop class (i.e., in the image of "suspension" $\left.\Omega: H^{+1}\left(\Omega^{-1} B, C\right) \rightarrow H(B, C)\right)$; thus $g$ is an $H$-map, and $D$ admits a multiplication. In fact, up to homotopy $D$ admits only one multiplication and $g$ only one homotopy multiplier $G$. Now, if $(\mathcal{O}, \mathcal{N}, j)$ is additive, $h$ is an $H$-map and $Z$ admits a multiplication. Since $\pi_{i}(D)=0$ for $i<q+1$ and $i>p+q, \psi$ must also be an $H$-map by Theorem 4 . Let $\Psi$ be the homotopy multiplier for $\psi$. Projecting into $\mathscr{P}(B)$ we obtain a homotopy multiplier $F$ for $f$. Projecting into $\mathscr{P}(\mathscr{L C})$ we obtain a map which looks like:

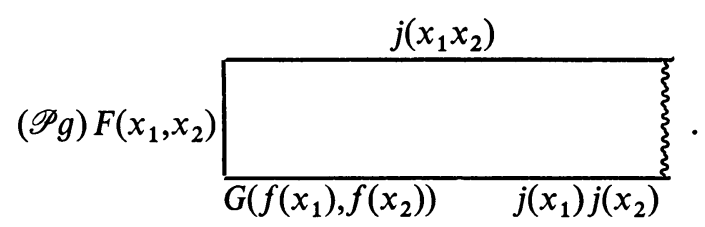

From this, it is easy to derive the required homotopy multiplier $J$. This completes Theorem 12.

Interpreting our results in cohomology we have:

Corollary 13. A space $Z$ is determined by cochains $u \in C^{q+1}(G, p ; H), v \in$ $C^{r+2}(H, q+1 ; K)$ and $w \in C^{r+1}(G, p ; K)$ such that $\delta w=v \circ u$. For $Z$ to admit a multiplication, it is sufficient that there exist cochains

and

$$
x \in C^{q}((G, p) \wedge(G, p), H), y \in C^{r+1}((H, q+1) \wedge(H, q+1), K)
$$

such that

$$
z \in C^{r}((G, p) \wedge(G, p), K)
$$

(1) $\delta x=\left\{m^{*}(u)\right\}$,

(2) $\delta y=\left\{n^{*}(v)\right\}$

(3) $\delta z=\left\{m^{*}(w)\right\}-v \circ x-y \circ(u \wedge u)$,

(where $\left\{m^{*}(u)\right\}$ is the component of $m^{*}(u)$ in $C^{q+1}((G, p) \wedge(G, p), H)$, etc.). The condition is necessary if $r<p+q$.

The complicated details of this corollary can be summarized by defining, in the category of $H$-spaces and $H$-maps, a secondary operation

$$
\begin{aligned}
& \mathscr{M}: \operatorname{Prim} H(A, B) \cap \operatorname{Ker} \mathscr{N} \rightarrow \\
& H^{-1}(A \wedge A ; C) /(\Omega \mathscr{N}) \circ H^{-1}(A \wedge A ; B)+\left\{m^{*}\right\} H^{-1}(A ; C) \\
& \quad+H^{-1}(B \wedge B ; C) \circ[u \wedge u] .
\end{aligned}
$$

This may perhaps be convenient for theoretical purposes, but it gives us no new information. 
4. Homotopy associative extensions. Now let us return to the multiplication constructed in Theorem 2. Even if $m$ and $n$ are associative, the multiplication $s$ need not be homotopy associative. To study this problem more thoroughly, we need some terminology.

Definition 14. A homotopy associative $H$-space $(X, m, Q)$ consists of an $H$-space $(X, m)$ and associating homotopy $Q: I \times X^{3} \rightarrow X$ such that

(0) $Q(0, x, y, z)=x(y z)$,

(1) $Q(1, x, y, z)=(x y) z$,

i.e., the diagram

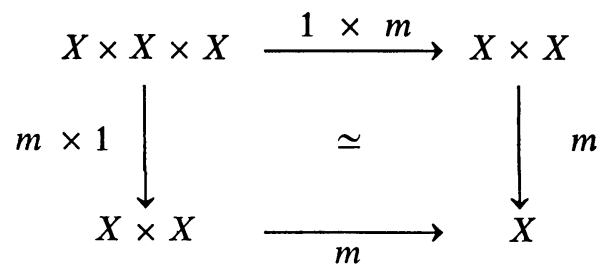

is homotopy commutative.

Maps of homotopy associative $H$-spaces are somewhat complicated to describe. Suppose that $(W, n, R)$ and $(Y, m, Q)$ are homotopy associative $H$-spaces and that $f:(X, n) \rightarrow(W, n)$ is an $H$-map. Given a sputnik homotopy between $n(f \times f)$ and $f m$, we can define a homotopy between $R \circ(1 \times f \times f \times f) \mid \dot{I} \times X^{3}$ and $f Q \mid \dot{I} \times X^{3}$. We can then ask if this homotopy can be extended to one between $R \circ(1 \times f \times f \times f)$ and $f Q$.

Definition 15. A map $f: Y \rightarrow X$ is a homotopy associative map of a homotopy associative $H$-space $(X, m, Q)$ into a homotopy associative $H$-space $(W, n, R)$ if

(1) there exists a sputnik homotopy $h_{i}: X \times X \rightarrow W$ such that $h_{0}\left(x, x^{\prime}\right)$ $=f(x) f\left(x^{\prime}\right)$ and $h_{1}\left(x, x^{\prime}\right)=f\left(x x^{\prime}\right)$ [i.e., $f$ is an $H$-map], and

(2) there exists a homotopy $d_{s}: I \times X^{3} \rightarrow W, 0 \leqq s \leqq 2$, such that

(b) $\quad d_{2}\left(t, x, x^{\prime}, x^{\prime \prime}\right)=f Q\left(t, x, x^{\prime}, x^{\prime \prime}\right)$,

$$
\begin{aligned}
d_{0}\left(t, x, x^{\prime}, x^{\prime \prime}\right) & =R\left(t, f(x), f\left(x^{\prime}\right), f\left(x^{\prime \prime}\right)\right), & & \\
d_{2}\left(t, x, x^{\prime}, x^{\prime \prime}\right) & =f Q\left(t, x, x^{\prime}, x^{\prime \prime}\right), & & \\
d_{s}\left(0, x, x^{\prime}, x^{\prime \prime}\right) & =f(x) h_{s}\left(x^{\prime}, x^{\prime \prime}\right), & & 0 \leqq s \leqq 1, \\
& =h_{s-1}\left(x, x^{\prime}, x^{\prime \prime}\right), & & 1 \leqq s \leqq 2, \\
d_{s}\left(1, x, x^{\prime}, x^{\prime \prime}\right) & =h_{s}\left(x, x^{\prime}\right) f\left(x^{\prime \prime}\right), & & 0 \leqq s \leqq 1, \\
& =h_{s-1}\left(x x^{\prime}, x^{\prime \prime}\right), & & 1 \leqq s \leqq 2 .
\end{aligned}
$$

Perhaps a picture would be helpful. Corresponding to $d_{s}$, we have a map of $[0,2] \times I$ into $W^{X^{3}}$ which for each point $\left(x, x^{\prime}, x^{\prime \prime}\right)$ can be depicted as follows: 


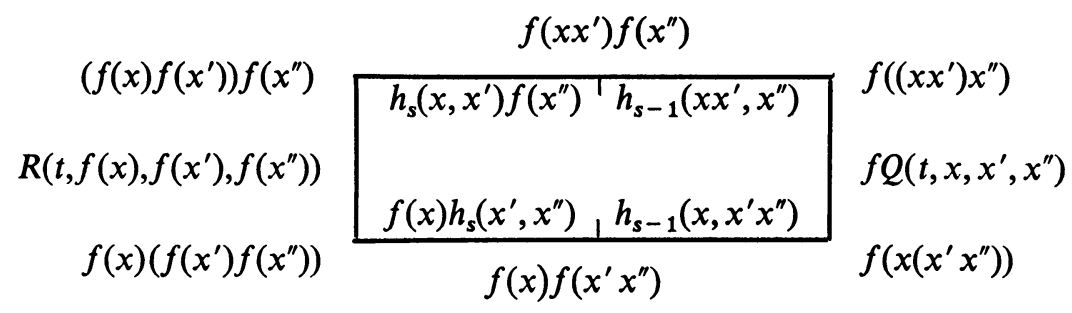

TheOREM 16. Let $(X, m, Q)$ and $(W, n, R)$ be homotopy associative H-spaces. Let $Y$ be as in Theorem 2. If $f: X \rightarrow W$ is a homotopy associative map, then there exists a homotopy associative multiplication on $Y$.

Proof. Let $f$ be a homotopy associative map with $h_{t}$ and $d_{s}$ as in Definition 15 . Let $s: Y \times Y \rightarrow Y$ be constructed using $h_{t}$ as in Theorem 2 . It is easy to see that $s(1 \times s)$ can be deformed to $\phi_{0}: Y^{3} \rightarrow Y$ given by

$$
\phi_{0}\left(y, y^{\prime}, y^{\prime \prime}\right)=\left(x\left(x^{\prime} x^{\prime \prime}\right), \lambda\left(\lambda^{\prime} \lambda^{\prime \prime}\right)+f(x) \cdot F\left(x^{\prime}, x^{\prime \prime}\right)+F\left(x, x^{\prime} x^{\prime \prime}\right)\right)
$$

for $y=(x, \lambda), y^{\prime}=\left(x^{\prime}, \lambda^{\prime}\right), y^{\prime \prime}=\left(x^{\prime \prime}, \lambda^{\prime \prime}\right)$, while $s(s \times 1)$ can be deformed to

$$
\phi_{1}\left(y, y^{\prime}, y^{\prime \prime}\right)=\left(\left(x x^{\prime}\right) x^{\prime \prime},\left(\lambda \lambda^{\prime}\right) \lambda^{\prime \prime}+F\left(x, x^{\prime}\right) \cdot f\left(x^{\prime \prime}\right)+F\left(x x^{\prime}, x^{\prime \prime}\right)\right) \text {. }
$$

Now corresponding to $d_{s}$ we construct a map $D: I \times X^{3} \rightarrow \mathscr{P}(W)$ in the usual way. An associating homotopy for $Y$ is given by means of

$$
\phi_{t}\left(y, y^{\prime} y^{\prime \prime}\right)=\left(Q\left(t, x, x^{\prime}, x^{\prime \prime}\right), \mathscr{L} R\left(t, \lambda, \lambda^{\prime}, \lambda^{\prime \prime}\right)+D\left(t, x, x^{\prime}, x^{\prime \prime}\right)\right) .
$$

As for $H$-maps, a converse theorem can be obtained in restricted situations. Since the same methods are used as for Theorem 3, we state only the results.

THEOREM 17. Let $Y$ be as in Theorem 2. Suppose that $f$ is an H-map with sputnik homotopy $h_{t}$ and that $s: Y \times Y \rightarrow Y$ is obtained from $h_{t}$ as in Theorem 2 . If there is an associating homotopy $S: I \times Y^{3} \rightarrow Y$ for s such that $\mathfrak{p} S\left(t, y_{1}, y_{2}, y_{3}\right)$ $=Q\left(t, \mathfrak{p}\left(y_{1}\right) \mathfrak{p}\left(y_{2}\right), \mathfrak{p}\left(y_{3}\right)\right)$, then $f$ is a homotopy associative map (with this $h_{t}$ as sputnik homotopy) under the following conditions: $X$ is $(p-1)$-connected and for some $q>p, \pi_{i}(W)=0$ for $i<q$ and $i>2 p+q$.

THEOREM 18. Let $Y$ be a space with only two nonvanishing homotopy groups $\pi_{p}(Y)=\pi$ and $\pi_{q}(Y)=G, q>p . Y$ admits a homotopy associative multiplication if and only if the $k$-invariant $k \in H^{q+1}(\pi, p ; G)$ is represented by some homotopy associative $\operatorname{map} f: K(\pi, p) \rightarrow K(G, q+1)$.

APPLICATION 19. Let $\alpha$ be a generator of $H^{n+1}\left(Z_{p}, n ; Z\right), n$ odd, $p$ an odd prime. The space with $k$-invariant $\alpha^{p}$ admits a multiplication but not one which is homotopy associative.

That $\alpha^{p}$ is primitive is easy since $m^{*}\left(\alpha^{p}\right)=\left(m^{*}(\alpha)\right)^{p}=\left(p_{1}^{*}(\alpha)+p_{2}^{*}(\alpha)\right)^{p}$ $=\Sigma\left(i_{1} p-i\right) p_{1}^{*}\left(\alpha^{i}\right) \otimes p_{2}^{*}\left(\alpha^{p-i}\right)=p_{1}^{*}\left(\alpha^{p}\right)+p_{2}^{*}\left(\alpha^{p}\right)$ because $\alpha$ is of order $p$ and $p$ 
divides $(i, p-i)$ for $1<i<p$. In [8] we perform the calculations necessary to show that $\alpha^{p}$ is not represented by a homotopy associative map. This requires some work, though basically it is a straightforward problem in obstruction theory. For $p=3$, it is a consequence of Moore's statement [6] that $\alpha^{p}$ is not in the image of "suspension".

$$
\sigma: H^{p(n+1)+1}\left(Z_{p}, n+1 ; Z\right) \rightarrow H^{p(n+1)}\left(Z_{p}, n ; Z\right) .
$$

\section{BIBLIOGRA PHY}

0. J. F. Adams, On the non-existence of elements of Hopf invariant one, Ann. of Math 72 (1960), 20-104.

1. J. F. Adams and P. J. Hilton, On the chain algebra of a loop space, Comment. Math. Helv. 30 (1956), 305-330.

2. A. H. Copeland, On H-spaces with two non-trivial homotopy groups, Proc. Amer. Math. Soc. 8 (1957), 184-191.

3. P. J. Hilton, Homotopy theory and duality (mimeographed), Cornell University, 1959.

4. I. M. James, On H-spaces and their homotopy groups, Quart. J. Math. Oxford. Ser. (2) 11 (1960), 161-179.

5. J. C. Moore, The double suspension and p-primary components of homotopy groups of spheres, Bol. Soc. Mat. Mexicana 1 (1956), 28-37.

6. - On the homology of $K(\pi, n)$, Proc. Nat. Acad. Sci. U.S.A. 43 (1957), 409-411.

7. - Seminario J. C. Moore (duplicated), Inst. de Matematicas de la Universidad Nacional Autonoma de Mexico, 1958.

8. J. D. Stasheff, Homotopy associativity of H-spaces. I, II, Trans. Amer. Math. Soc. (to appear).

Massachusetts Institute of Technology,

CAmbridge, MassachusetTs 